\title{
Construcción de la propia paternidad en adolescentes varones pertenecientes a liceos municipales de la comuna de La Cisterna ${ }^{1}$
}

Construction of the self paternity in male teenagers who
belong to municipal high schools of La Cisterna commune

Cecilia Anabalón ${ }^{2}$

Francisca Cares ${ }^{3}$

Ricardo Cortés ${ }^{4}$

Macarena Zamora ${ }^{5}$

Resumen

Esta investigación tuvo como objetivo conocer la articulación entre los elementos de los ámbitos social, familiar y escolar en la construcción de la propia paternidad, en adolescentes varones pertenecientes a liceos municipales de la comuna de La Cisterna (Santiago, Chile). El estudio es de carácter cualitativo centrado en la fenomenología, desde una perspectiva hermenéutica, siendo la técnica de recolección de datos la entrevista en profundidad. Se obtuvo como resultado principal que el área más significativa para la construcción de la paternidad es la familia y como figura antagónica identificaron al grupo de pares. A la vez todos los resultados de esta investigación dan cuenta del surgimiento de una nueva paternidad.

Palabras Clave: masculinidad, paternidad y adolescencia.

$1 \quad$ Basado en la tesis para obtener el Grado de Licenciados en Psicología. Universidad Católica Silva Henríquez. Santiago, Chile. Profesor Guía Dr. en Psicología Héctor Cavieres Higuera. cpaternidad@gmail.com

2 Cecilia Anabalón Armijo, Psicóloga de la Universidad Católica Silva Henríquez. E-mail cecilia. anabalon@gmail.com

3 Francisca Cares Pozo, Psicóloga de la Universidad Católica Silva Henríquez. E-mail cares.fran@ hotmail.com

4 Ricardo Cortés Belmar, Psicólogo de la Universidad Católica Silva Henríquez. E-mail ps.rcortes@ gmail.com

5 Macarena Zamora Contardo, Psicóloga de la Universidad Católica Silva Henríquez. E-mail ps.macazamora@gmail.com 


\begin{abstract}
This research's aim is to understand the linkages between the elements of social context, family and school in the construction of one's parenting, in male adolescents who belong to public high schools in the district of La Cisterna. This qualitative study is focused on phenomenology, hermeneutics perspective, and in-depth interviews as the technique of data collection. The main result was that the most significant area for the construction of fatherhood is family, and as an antagonic area, they identified the figure of the peer group. Also, all the results of this study report the emergence of a new fatherhood.
\end{abstract}

Key words: masculinity, paternity and adolescence. 


\section{Introducción}

Los hombres han incursionado de manera más afectiva en la paternidad, sin embargo continúan conservando algo de distancia, lo que es propio de la caracterización de su género. Este distanciamiento puede ser aún más complejo de acuerdo al contexto en el cual la paternidad surge, como por ejemplo en la adolescencia, donde la brecha entre los varones adolescentes y su función paterna se acrecienta (Madrid \& Olavarría, 2005).

Ser padre adolescente es un fenómeno social de gran relevancia, pues es un hecho que marca la vida de sus protagonistas y la de sus familias, ya que si bien la paternidad puede considerarse como parte del ciclo vital de los seres humanos, se presenta en una etapa no esperada. Lo anterior lleva a los jóvenes a enfrentarse a responsabilidades propias de un adulto y a un modelo de paternidad frente al cual, generalmente, no tienen posibilidades para resolver sus deberes (Madrid \& Olavarría, 2005).

Hoy en día, tanto la función maternal como la paternal se están adelantando, puesto que son cada vez más los casos de paternidad a temprana edad. Esto se puede observar mediante estadísticas arrojadas por el censo de población efectuado en el año 2000 en Chile, donde existía un $5,1 \%$ de paternidades en adolescentes menores de 20 años, mostrando un aumento de un $200 \%$ en comparación a 1960 , donde se observaba solo un 1,7\% (Madrid \& Olavarría, 2005).

Este aumento de embarazos en la adolescencia, según Issler (2001) se debe a múltiples factores predisponentes, como la madurez reproductiva temprana, el inicio precoz de las relaciones sexuales, el poseer una familia disfuncional, el bajo nivel educativo, las fantasías de esterilidad, la falta de información y el cambio cultural, entre otros.

Según los datos arrojados por el Instituto Nacional de Estadísticas de los 39.902 nacimientos en 2008 cuyas madres son adolescentes, hubo 12.148 casos en que el padre también es menor de 20 años, lo que significó que del total de nacimientos provenientes de madres menores de 20 años, casi un tercio tienen madre y padre adolescente (INE, 2010).

La presente investigación se enfocó en los varones que son padres en la adolescencia, cuestión que resulta interesante debido a que, por 
lo general, el problema tiende a feminizarse, lo que da cuenta de una tendencia social a relacionar el embarazo y sus eventuales problemáticas, únicamente con la mujer (Cruzat $\&$ Aracena, 2006). Es por esto, que es relevante conocer cómo los adolescentes varones construyen su paternidad y qué elementos psicosociales están a la base de dicha construcción, tales como la masculinidad, el género y la adolescencia, entre otros.

El desarrollo de las relaciones significativas en el período de la adolescencia está delimitado por los contextos y ámbitos que son propios de esta etapa de la vida. Los adolescentes se desenvuelven principalmente en tres ámbitos: la familia, la escuela y lo social, (este último referido al grupo de pares) (Konterllnik, 1998, citado en Hamel, Luco \& Osses, 2000). Son estos tres ámbitos en los cuales se fundamentó esta investigación a fin de observar cómo influyen en la generación de significados sobre la paternidad.

Bajo el marco de esta investigación surgió como principal interrogante, ¿cuál es la construcción de la propia paternidad en adolescentes varones pertenecientes a liceos municipales de la comuna de La Cisterna?, además de ¡cuál es la construcción de esta paternidad en relación a su ámbito familiar, escolar, y social?

\section{Marco Teórico}

Desde la perspectiva de género, la construcción del ser hombre y ser mujer es el resultado de un conjunto de procesos relacionales, de intercambios de carácter entre personas y el mundo material, que producen y reproducen significados, dando sentido a las acciones por medio de esta interacción (Gergen, 2001, citado en Gutiérrez, 2006).

Desde Burin y Meler (1998), el género posee tres rasgos fundamentales que lo caracterizan: es siempre relacional, tiene su base en una construcción histórico-social y posee aspectos determinantes de la subjetividad humana. Desde esta base teórica, se puede comprender que el sujeto introyecta las pautas socializadoras, con las cuales construye su propia masculinidad, a través de la interacción con su entorno, adquiriendo 
de esta manera las pautas de comportamiento que le permiten actuar como varón y ser reconocido como tal.

Por su parte, Stoller (1968, citado en Lamas, s/f) señala que el género se compone de tres instancias básicas: la asignación de género, que se establece por una diferenciación sexual; la identidad de género, en donde se produce una estructuración de la experiencia vital a partir de la asignación de su propio género; y finalmente, el papel del género, el cual se configura con el conjunto de normas y prescripciones que dictan la sociedad y la cultura sobre el comportamiento femenino o masculino. En este último punto se centró esta investigación a fin de observar cómo se articula este rol en el marco de la interacción con el medio.

Desde esta perspectiva, lo masculino lo entenderemos como una construcción social de roles que se asignan según la condición biológica que se posea. En este marco es que las relaciones de género se desarrollan dentro de la división masculino - femenino, la que actúa como un mecanismo de división cultural que organiza y da sentido a las prácticas sociales. Por lo tanto, la masculinidad puede ser entendida como una práctica social que se construye definiéndose en función de ciertos repertorios ideológicos o culturales socialmente compartidos (Alvarado, 2005).

Las características que son socialmente atribuidas al papel de cada género se consideran mandatos. Según Hamel, Luco y Osses (2000) estos se definen como creencias culturales respecto del comportamiento de las personas según su rol, que son históricamente construidas y atribuidas a los individuos en su papel genérico.

En el caso de los varones se espera que el papel en la familia sea principalmente el de proveedor económico, por ende el hombre que trate de equilibrar sus intereses con la responsabilidad hacia la crianza de los hijos o que acepte compartir la manutención con su pareja, iría en contra de lo esperado según el mandato cultural. Lo anterior da cuenta de que estas situaciones limitan socialmente la manera en que los adolescentes construyen su concepción de masculinidad (Hamel, et al. 2000).

Es necesario considerar la paternidad como un proceso de construcción, lo que supone una comprensión que tiene como resultado una tarea de interrelación entre las personas. Esta forma de comprender el 
medio prevalece a través del tiempo mediante la validez empírica que el sujeto le otorgue a sus procesos.

Alatorre y Luna (2000) señalan que la paternidad se construye por medio de los procesos socioculturales y subjetivos que dan lugar a las prácticas y significaciones en relación con los hijos e hijas. En el caso de los adolescentes, se construye con base en sus ámbitos de desarrollo y de las relaciones que vayan generando.

Ser padre adolescente, implica considerar tanto el concepto de masculinidad como la mirada que la sociedad atribuye al ser padre adolescente. Se da por sentado muchas veces, que las posiciones masculinas son representaciones neutras de valores y prácticas humanas universales, impuestas a la fuerza como marcos de convivencia social (Szil, 2007).

Los roles asignados a los diferentes sexos son aprendidos desde la primera infancia y se manifiestan de manera longitudinal en nuestras vidas. Se espera que los hombres asuman ser los procreadores, mientras que encargarse de la protección y el mantenimiento de los hijos es algo asignado a las mujeres. Esta diferencia es algo construido socialmente no inherente al ser humano (Szil, 2007).

Las concepciones de masculinidad y de subjetividad como construcciones simbólicas y de interpretaciones disponibles a las instituciones que las regulan permiten abordar la paternidad como un fenómeno cultural, social y subjetivo, de enorme diversidad dentro del mismo individuo, entre los individuos de un mismo contexto sociocultural y en diferentes momentos históricos. Es decir, esta concepción será propia de cada padre, dependiendo de su entorno e influencia (Castillo \& Centeno, 2005). Por lo tanto, el fenómeno de la paternidad está condicionado por las pautas de interacción social en las que el sujeto se encuentra, de tal manera que el adolescente construirá su propia concepción de la paternidad.

Por último y en el marco de un enfoque de género que sustenta cierta visión de la masculinidad, Cardoso (1998, citado en Viveros, 2000) sostiene la existencia de un "muro de silencio" que rodea la paternidad adolescente, y la divide y desplaza, implicando una relación perversa de la sociedad con el joven padre. $\mathrm{Al}$ anular socialmente este tipo de paternidad, 
se acaba por legitimar la ausencia paterna, pues se le dificulta al adolescente la posibilidad de pensar, prevenir o asumir su condición de padre.

Lo anterior crea una relación dispersa entre el adolescente y la sociedad, motivando a que los adolescentes se sientan vulnerables ante su nueva condición, limitando su capacidad de pensar, prevenir y/o asumir su rol de cuidador y protector durante la paternidad (Hernández, Quevedo \& Ramos, 2008).

\section{Metodología y procedimientos}

El presente estudio tuvo como objetivo conocer la articulación de los elementos de los ámbitos familiar, escolar y social en la construcción de la propia paternidad, en adolescentes varones pertenecientes a liceos municipales de la comuna de La Cisterna. La investigación es de carácter cualitativo, definido por Sandoval (1996) como una ruta metodológica que se relaciona más con el descubrimiento y el hallazgo que con la comprobación o la verificación.

El fundamento teórico se basó en la fenomenología, la cual trata de describir la experiencia sin acudir a explicaciones causales, permitiendo establecer un contacto directo con el fenómeno estudiado (Sandoval, 1996).

\section{Definición de muestra}

Se utilizó una muestra intencional de caso típico, ya que para lograr el propósito de esta investigación era necesario identificar adolescentes bajo los siguientes criterios de inclusión: varones, debido a que es desde esta perspectiva que se ha enfocado este estudio; cuya edad fluctúe entre los 15 a 20 años, período propio de la adolescencia; que hayan sido padres; que pertenezcan a los liceos municipales de la comuna de La Cisterna, por encontrarse entre las tres comunas con mayor número de alumnos padres y madres matriculados en liceos municipales de la Región Metropolitana (Olavarría, 2006). Además, la participación de ellos debía ser voluntaria. Se accedió al objeto de estudio gracias a la colaboración de los directivos de los establecimientos. 
En términos de procedimiento los liceos fueron contactados vía Dirección Municipal de Educación. Una vez en ellos y con autorización de la dirección de los establecimientos, se entrevistó a los orientadores y jefes de la Unidad Técnica Pedagógica, quienes entregaron la información acerca de los casos de paternidad adolescentes conocidos en el establecimiento. Luego se contactó con los jóvenes y se les invitó a participar de la investigación, los que en suma fueron siete estudiantes.

Respecto de las consideraciones éticas, se solicitó el respaldo de los liceos y la firma de una carta de consentimiento informado por parte de los estudiantes y de sus padres, instancia en la cual los investigadores comprometieron la confidencialidad en el tratamiento de la información.

\section{Técnicas de recolección de datos}

La técnica de recolección de datos utilizada en esta investigación fue la entrevista en profundidad, la cual implica una sumersión del entrevistado con el entrevistador. A través de ella se obtuvieron relatos de experiencias destacadas de la vida de los entrevistados y las definiciones que ellos le otorgan al acontecimiento de ser padre a temprana edad (Sandoval, 1996). Las entrevistas fueron realizadas por dos investigadores y la duración de ellas fue de aproximadamente una hora en las dependencias de los establecimientos dispuestos para dicho propósito.

\section{Procedimiento de análisis de datos}

Se utilizó la técnica de análisis de contenido, para lo cual se trascribieron las entrevistas y luego se levantaron categorías desde los elementos más reiterativos en el relato. Lo anterior permitió realizar una lectura de la realidad social, las acciones humanas y la cultura, a la manera de textos sobre los cuales es posible emprender un trabajo de tipo hermenéutico (Sandoval, 1996).

\section{Resultados}

La construcción es una tarea cooperativa y activa que emerge debido a las relaciones que se establecen con los otros. En la adolescencia, 
los principales ámbitos de interacción son la familia, la escuela y los amigos, y dentro de ellos surgen ciertos elementos que coarticulan la construcción de la realidad. En los casos de paternidad adolescente identificados en los liceos municipales de la comuna de La Cisterna, se observan elementos significativos que los adolescentes integran en la construcción de su paternidad y que definen la manera en que actúan como padres.

En el ámbito familiar se evidenciaron tres elementos centrales que son la figura del padre, la figura de la madre y la figura de los hermanos.

Respecto al padre se puede mencionar que este se liga a características asociadas a la enseñanza, lo cual queda claro en el relato de A.N. que señala: "Enseñarle cosas, que aprenda... mi papá siempre nos sacaba... igual trato de llevar el mismo ejemplo que ellos". De este extracto se infiere que para A.N. la educación es algo importante para el desarrollo de las personas y se atribuye la responsabilidad a los padres de educar a los hijos. Respecto de la figura paterna se reconoce que las relaciones que se establecen entre padre e hijo son cercanas. H.M. da cuenta de esto al referirse a su padre como un amigo: "Ah, como mi papá, porque somos como amigos. Supongamos que si lo necesito, él va y me ayuda". Lo anterior hace referencia a la idea de un padre presente, porque H.M. tiene la certeza de que su padre lo apoyará de manera comprensiva frente a las situaciones complejas. Los jóvenes tienden a recurrir más al padre que a la madre, a pesar de que la comunicación con él es, en muchos casos, precaria. Esto se puede observar en el siguiente relato de L.O., quien dice: "La relación con mi papá es buena, pero mi papá no habla mucho, hay que sacarle las palabras, pero igual sentí más apoyo de él". Desde esta afirmación se puede señalar que a pesar de no tener una comunicación fluida con el padre, logran mantener conversaciones que son influyentes para el adolescente.

Por lo anterior, se deduce que la figura paterna es una figura significativa para los adolescentes al momento de construir su propia paternidad, de ella se rescatan principalmente relatos en los que se reflejan conceptos como ayuda, educación y protección, entre otros. Es posible señalar del análisis de estos relatos que la figura más relevante en la construcción de la paternidad es precisamente el propio padre, aún cuando 
este no sea con quien se tiene más confianza o se dialogue con mayor habitualidad. Lo anterior se condice con lo que Olavarría (2001) refiere, dando cuenta de que el aprendizaje de la paternidad se articula a través de la internalización de su figura paterna en la vida cotidiana, ya sea mediante la imitación o diferenciación.

En cuanto a la madre, los adolescentes incorporan elementos para la construcción de su propia paternidad, tales como la lucha frente a la adversidad y su abnegación en la crianza. Esto se refleja en el relato de F.Q., quien nos dice: "No sé... mi mamá no es tan joven, pero siempre tuvo que estar luchando por mi y por mi hermano, por la mala suerte que se van po', igual ha luchado caleta por nosotros... Por lo mismo yo necesito terminar el cuarto, porque mi mamá me dijo: 'no me vai a defraudar otra vez', porque mi hermano no lo terminó. Y yo con la esperanza sipo, igual lo hice por el niño, porque nunca tengo que dejarlo solo; aunque termine la relación con mi pareja yo tengo que seguir con él po', si... es mi hijo". Se deduce la existencia de un sentimiento de compromiso con los hijos, ya que el adolescente manifiesta una preocupación y un deseo de mantener una relación constante y presente con su hijo a lo largo del tiempo, puesto que reconoce en su madre ciertos valores de cuidado y de lucha por la familia. Esto también se refleja en el relato de L.O., donde afirma: "Ella es preocupada de todo, se preocupa de mis estudios, que no me falte nada, de lo que me pasa, si me ve triste, me pregunta y si me ve enojado me deja solo pa' que se me pase". Se puede inferir de lo anterior que el adolescente entiende que la contribución de la madre en la construcción de la paternidad no solo está relacionada a los aspectos más emocionales, sino que también esto implica una acción, lo que se refleja en la necesidad de los adolescentes por hacer algo para sus hijos.

Lo anterior da cuenta de una figura materna tradicional, asociada a la abnegación por los hijos, al cuidado, al sacrificio. Lo interesante es advertir que los varones adolescentes rescatan estas características propias de lo femenino para la construcción de su propia paternidad, lo que da paso a una nueva paternidad. Esto es análogo a lo mencionado por Gallardo, Gómez, Muñoz y Suárez (2006) quienes refieren que las representaciones tradicionales de la paternidad inscritas a lo largo de la 
historia como un opuesto al referente femenino se han visto trastocadas por diversos contextos socioculturales. Lo mencionado se refleja en el actual replanteamiento y transformación de los roles masculinos, los cuales se expresan de manera más crítica en los jóvenes urbanos y con mayor escolaridad.

Referente a los hermanos, en los casos en que estos ya han sido padres, son considerados por los entrevistados como un apoyo cercano. Esta relación fraternal se caracteriza por el compañerismo, admiración e incluso complicidad. Por ejemplo, R.Z. dice: "Yo se lo dije junto con $m i$ hermano, porque fuimos papás a la misma vez, mi hermano va a cumplir 20 años y nuestros hijos se llevan por 24 dias nomás". Se observa que la relación entre hermanos es horizontal, debido a que se apoyan siendo ambos padres en circunstancias similares. También acerca de los hermanos R.Z. dice lo siguiente: "Responsable, es más responsable que yo, porque él tiene un buen trabajo, él igual es súper preocupado por su hijo, es un buen ejemplo, además es mejor que esté toda la familia junta, que estar separado". Cabe destacar que esta situación se presenta solo en este caso en particular, pero es significativo para la construcción de la paternidad del adolescente, pues se identifica con su hermano.

Por otro lado, en el ámbito escolar emergen dos referentes centrales, los cuales corresponden al orientador escolar y a los compañeros de curso.

Respecto del orientador, se puede indicar que para los adolescentes esta figura se presenta con más relevancia que sus docentes, ya que son quienes, en ciertos establecimientos, se responsabilizan de la situación del joven, a través del acompañamiento y el consejo. Esto se evidencia en el relato de L.O., quien afirma: "El orientador me apoya harto, (...) es como un apoyo que siento por los problemas, si tengo un problema en el colegio no le cuento a mis papás, pero voy donde él". A pesar de lo anterior, se advierte la falta de apoyo y los sentimientos de desamparo que refieren sentir por parte de las instituciones educativas. Este respaldo o apoyo es distinto al entregado a las adolescentes en las escuelas. Tal como lo menciona Cruzat y Aracena (2006) quienes especifican que los adolescentes no son respaldados, existiendo menos preocupación que la que reciben las adolecentes en estas mismas circunstancias. 
Relativo a los compañeros de curso que ya han sido padres, se establecen relaciones que dan cuenta de interacciones cercanas en las cuales se intercambian experiencias con compañeros en la misma condición de paternidad y otras donde reflexiona respecto del hecho de ser padre, F.Q. afirma: 'Si po', conversamos de las guaguas que están grandes y todo... somos dos parejas en el curso, conversamos, grabamos sus videos y nos mostramos... ah! mi hijo hace lo mismo". Esto demuestra una conexión mayor por estar ambas parejas en la misma situación de ser padre en la adolescencia. En una segunda instancia, se puede decir que el adolescente identifica aspectos negativos en algunos compañeros, A.N. menciona: "Mm... nada, es que algunos no son responsables, o sea, no le toman el peso a tener un hijo". Esto da cuenta nuevamente de una construcción de paternidad adolescente por contraste.

Por último, en el ámbito social se evidenció como elemento central al grupo de pares. Es preciso señalar que este ámbito es relevante debido a la necesidad de considerar la cultura de los adolescentes padres, conocer su visión de mundo, sus propias concepciones, sin apartar que la masculinidad responde a la construcción social del ser hombre dentro de una sociedad y cultura determinada (Castillo \& Centeno, 2005).

Los jóvenes identifican aspectos que no quisieran repetir en su experiencia, lo que da cuenta que su construcción de paternidad no es solo imitar aspectos de otros, sino que también de lo que no les gustaría hacer. Un ejemplo de esto se extrae del relato de R.Z. quien dice: "No los veo mucho como un ejemplo, pero si me sirve para saber que yo no voy a hacer esas cosas con mi hijo". Si bien la relación entre amigos se puede considerar como algo significativo, se vuelve algo contradictorio respecto de lo que ellos quieren hacer como padres. Esto también se refleja en el relato de A.N., quien señala: "Sí po', antes yo salía todos los fines de semana, ahora puro estudio, trabajo y ella no más (hija), los amigos quedaron de lado. Igual hay varios que tienen hijos, pero siguen saliendo y todo eso...em, no sé po', los veo como irresponsables, no los veo como ejemplo, porque se puede ver que han hecho cosas malas, entonces los veo como ejemplos de lo que no hay que hacer. En el relato precedente es aún más explícita la tendencia mencionada anteriormente. 


\section{Conclusiones}

Considerando los antecedentes revisados es posible mencionar que es difícil encontrar elementos específicos que aborden la temática de la paternidad adolescente. Se puede señalar más bien que la sociedad, aún en los tiempos actuales, valida roles donde el hombre se encuentra menos integrado en el proceso de paternidad, considerándosele de un modo más distante y ajeno a esta vivencia (Cruzat $\&$ Aracena, 2006). Sin embargo, dado el carácter de construcción del proceso de la paternidad, es posible indicar que la definición de las conductas asociadas a la función paterna pueden cambiar no solo con el transcurso de los años, sino que además existen diferencias de una cultura a otra, o de una sociedad a otra e incluso entre grupos, etnias o clases pertenecientes a un mismo contexto. Así entonces, la construcción de la paternidad toma no solo elementos del contexto cultural y el entorno en el cual se desarrolle el adolescente, sino que además influirán las relaciones familiares y cercanas que este establezca en ese momento. A partir de este razonamiento es que esta investigación buscó indagar en los elementos de los ámbitos familiar, escolar y de grupos de pares por considerarse que estos abarcan gran parte de las relaciones sociales que un adolescente establece (INJUV, 2006).

En la presente investigación, a partir de las entrevistas realizadas, se observó que incluso en un mismo sector sociocultural, como lo es la comuna de La Cisterna, surgen diferencias en la construcción de la paternidad, cuestión que apoya el carácter de construcción psicosocial de la misma.

Respecto de los elementos comunes que aparecen en los discursos de los jóvenes, es relevante destacar la importancia de las relaciones familiares y en particular del vínculo establecido con el padre en dicha construcción. A partir de los antecedentes recopilados se puede señalar que los adolescentes extraen los conceptos de compañia, amistad y apoyo, asociados a la figura del padre, mientras que en la figura de la madre, se encuentra un referente de rigurosidad, esfuerzo y experiencia, conceptos que según los adolescentes entrevistados, integran y pretenden imitar en su 
labor como padres, instalándose entonces como nuevas pautas de crianza en la relación de paternidad del nuevo padre adolescente.

Es posible entonces afirmar que para la construcción de paternidad adolescente es clave la imagen que el joven toma del padre, sin embargo a esta imagen el sujeto integra elementos que son socialmente asignados al género femenino, y es entonces esta integración la que da como resultado el surgimiento de una nueva paternidad.

Esta nueva paternidad se caracterizaría por una concepción en donde los adolescentes presentan mayor compromiso emocional y afectivo, más presencia en la crianza y en el desarrollo de los hijos. En esta particular construcción de su propia paternidad, los adolescentes contribuyen a seguir generando el cambio que se ha venido manifestando en el tiempo, aún cuando mantengan algunos aspectos ligados al modelo antiguo de padre como por ejemplo, el de sostenedor económico. El varón adolescente y su paternidad se encuentran cada vez menos ocultos tras lo que Cardoso (1998, citado en Viveros, 2000) denomina el "muro de silencio" que invalida su relación paternal.

En marcos socioculturales este proceso de transformación ha sido denominado como mutación cultural (Bajoit, 1995, citado en Sandoval, 2006). Este concepto refuerza la idea de que los jóvenes son los agentes de cambio que integran partes de los modelos antiguos en la transición con las nuevas tendencias. En este caso se marcaría la transición de un modelo patriarcal clásico a uno donde se amplíen los roles del padre a partir de la integración de aspectos históricamente asociados al género femenino.

Los resultados de esta investigación proyectan líneas de análisis y desafíos en torno a la intervención en la temática de la paternidad adolescente, dadas ciertas paradojas o contradicciones posibles de apreciar en el relato de los entrevistados.

Por ejemplo, los adolecentes erigen gran parte de su propia paternidad desde la relación con sus padres. Sin embargo también señalan que esta se da en el marco de conversaciones caracterizadas por su "deficiente fluidez". De este resultado se desprende, entonces, el requerimiento de que junto con generar estrategias de apoyo a los propios adolescentes sería necesario gene- 
rarlas para los padres de los mismos. De esta forma se podría ayudar a los padres de los adolescentes al desarrollo de competencias y mediaciones para apoyar su importante rol en el proceso de sus hijos, cuestión que además favorecería el vínculo entre ellos. Lo anterior a su vez podría ser considerado como un elemento relevante en la construcción de la paternidad del adolescente, tomando como parámetro una relación más cercana.

En relación al ámbito escolar es de suma importancia la constatación de que los adolescentes no se sienten apoyados por los establecimientos educacionales a los cuales asisten. Indican que no existen figuras significativas que se relacionen de manera constante con ellos respecto a esta nueva vivencia de ser padres. A pesar de que se hace mención al orientador(a), este apoyo es percibido como insuficiente, no cubriendo de manera adecuada las necesidades planteadas por los estudiantes. Aún cuando en los establecimientos se tiene conocimiento formal de los casos de los alumnos que son padres, se realizan acciones de apoyo mínimas.

Dado que el colegio es un lugar donde los adolescentes pasan gran parte del día compartiendo un mismo espacio físico, incluso más que con sus propias familias, es que se considera necesaria la generación de una línea de acción en donde psicólogos y/o orientadores trabajen con los adolescentes en un acompańamiento del proceso de paternidad, brindándoles apoyo emocional, estrategias de enfrentamiento al cambio y principalmente orientación con respecto a la toma de decisiones, ya que es en este punto en donde los jóvenes se sienten indefensos. Cuestiones de este tipo podrían aminorar la sensación de desamparo que manifiestan sentir. Lo anterior es coherente con lo planteado por Cruzat y Aracena (2006), quienes señalan que los jóvenes poseen un sentimiento de desorientación y de soledad al enfrentar su propia paternidad, ante lo cual requieren sentirse apoyados por su entorno social, educativo y familiar.

Ahora bien, no resultaría correcto dar toda la responsabilidad a los establecimientos educacionales, pues el problema en rigor está a nivel de la inexistencia de una política pública específica para el apoyo psicosocial de los adolescentes varones en nuestro país, ya que las legislaciones en toda Latinoamérica siguen excluyendo a los hombres como padres, generando 
una barrera que no les permite participar de programas que pudiesen derivarse de estas políticas, invalidando el desarrollo de su paternidad (Gallardo et al., 2006).

Lo anterior da cuenta que las instituciones sociales mantienen los mandatos culturales más arcaicos, dificultando que los adolescentes se sientan motivados a participar del proceso de ser padres y de asumir un rol activo en la crianza de sus hijos. Si se hace el ejercicio de mirar de manera extrema la limitación que realizan los establecimientos educacionales, puede significar para el adolescente la exclusión total de todo el proceso, ya que se legitima la ausencia paterna y se le dificulta pensar o asumir su nueva condición de padre.

Por último, según la propuesta del estudio junto a la familia y el colegio, el grupo de pares se constituye en un referente importante al momento de la construcción de la propia paternidad.

Los análisis en este ámbito, contrario de lo que se pudiera suponer, muestran que los adolescentes no hablan de su experiencia de ser padres con su grupo de amigos, creen tener un apoyo pero del cual es muy poco o casi nada lo que se concretiza. Esto es aún más acentuado en la relación de amigos donde ambos han sido padres, el tema pareciera ser un tabú, en donde no se opina de cómo experimentan la paternidad cada uno, sino que se tratan aspectos más triviales. Los adolescentes entrevistados observan la paternidad del otro como algo lejano y externo a lo que ellos vivencian. Incluso la mayoría de los entrevistados identificaba en los amigos que ya habían sido padres aspectos que ellos no quisieran replicar, siendo incapaces de hablarlo con ellos. Pareciera en este sentido que aparecen nuevos resabios de modelos de masculinidad más arcaicos donde entre los "hombres" no resulta pertinente conversar sobre cuestiones más profundas o relacionadas con ámbitos más íntimos o afectivos.

Es posible señalar que existen variados elementos que coarticulan la construcción de la paternidad de los adolescentes, por lo tanto se debe abarcar esta discusión de manera flexible y recoger antecedentes desde lo subjetivo y a la vez, observar cómo estas distintas realidades se integran en una sociedad cambiante que propone nuevos desafíos y presenta transformaciones culturales constantes. 
Si bien se han dado pasos importantes para la inclusión de los hombres en la función de la paternidad, estos aún se ven ocultos por aspectos culturales que se encuentran a la base de nuestra sociedad. Como lo expresa Gallardo et al. (2006) la sobrevaloración del rol materno resulta ser un impedimento para que el padre adolescente asuma otras funciones respecto de la crianza de los hijos. Este aspecto es complejo de solucionar y en él tienen un rol preponderante las propias madres de los adolescentes y la flexibilización de las instituciones sociales.

Es particularmente importante dar cabida a los aspectos subjetivos de la vivencia de ser padre, cuestión que se traduciría en la relativización de los mandatos culturales hacia la paternidad. Sin embargo, es posible decir que pareciera ocurrir al revés y que mediatizado por la influencia de las instituciones sociales lo que ocurre es que se tienden a acentuar los mandatos culturales clásicos.

Es preciso ser conscientes de que la transformación de una cuestión como la significación de la paternidad es muy difícil de generar. Se espera con este trabajo apreciar el problema de la paternidad adolescente y la configuración de la función paternal, a fin de que estos elementos pudiesen servir de base para futuras investigaciones y eventualmente tomar en cuenta estos aspectos en el abordaje del fenómeno, especialmente desde los contextos escolares. 


\section{Referencias}

Alatorre, J. \& Luna, R. (2000). Significados y prácticas de la paternidad en la Ciudad de México. En N. Fuller (Ed.), Paternidades en América Latina, pp. 241-275. Lima: Pontificia Universidad Católica del Perú, Fondo Editorial.

Alvarado, C. (2005). El laberinto de las masculinidades: de seminaristas, adolescentes pobres y estrellas juveniles del fútbol. Relato de tres experiencias de trabajo con adolescentes varones desde la salud sexual y reproductiva. Revista Adolescencia y Salud, 6(1-2)1-13. Obtenido el 20 de abril de 2009 desde http://www.binasss.sa.cr/revistas/ays/6n1/art4.pdf

Burin, M. \& Meler, I. (1998). Género y familia. Poder, amor y sexualidad en la construcción de la subjetividad. Buenos Aires: Ed. Paidós.

Castillo, M. \& Centeno, R. (2005). Masculinidad y factores socioculturales asociados a la paternidad. Estudio en Nicaragua. UNFPA-CEPAL. Obtenido el 20 de enero de 2011 desde http://www.berdingune.euskadi.net/u89-congizon/es/contenidos/informacion/material/es_gizonduz/adjuntos/Masculindad\%20y\%20Paternidad\%20Responsable\%20Nicaragua.Marcelina\%20Castillo\%20Venerio,Rebeca \%20 Centeno\%20Orozco.pdf

Cruzat, C. \& Aracena, M. (2006). Significado de la paternidad en adolescentes varones del sector sur-oriente de Santiago. Revista Psykhe, 15(1), 19-44. Obtenido el 20 de diciembre de 2010 desde http://www.scielo. cl/scielo.php?pid=S0718-22282006000100003\&script=sci_arttext

Gallardo, G., Gómez, E., Muñoz, M. \& Suárez, N. (2006). Paternidad: Representaciones Sociales en Jóvenes Varones Heterosexuales Universitarios sin Hijos. Revista Psykhe, 15(2), 105-116. Obtenido el 20 de enero de 2011 desde http://www.scielo.cl/scielo.php?pid=S071822282006000200010\&script $=$ sci_arttext

García, M., Ibáńez, J. \& Alvira, F. (2004). El análisis de la realidad social. Métodos y técnicas de investigación. Madrid: Ed. Alianza.

Gutiérrez, S. (2006). Género y masculinidad: relaciones y prácticas culturales. Revista de Ciencias Sociales, 111-112, 155-175. Obtenido el 15 de Abril de 2009 desde http://www.vinv.ucr.ac.cr/latindex/rcs004/revsoc-111-12.pdf

Hamel, P., Luco, A. \& Osses, G. (2000). Rock and roles masculinos: veamos las cosas de otra manera. CD ROM. Santiago: Aprofa 2000.

Hernández, J. Quevedo, D. \& Ramos, A. (2008). Vivencias de adolescentes varones frente al embarazo en un municipio del departamento de Risaralda. Trabajo de grado para optar al título de enfermera. Facultad de enfermería. Pontificia Universidad Javeriana. Bogotá. Obtenido el 10 de noviembre de 2009 desde http://www.javeriana.edu.co/biblos/tesis/enfermeria/tesis13.pdf 
Instituto Nacional de Estadísticas (2010). Estadísticas vitales. Informe anual 2008. Obtenido el 14 de enero de 2011 desde http://www. ine.cl/canales/menu/publicaciones/calendario_de_publicaciones/ pdf/21_12_10/vit_08211210.pdf

Instituto Nacional de la Juventud (2006). 5ta Encuesta Nacional de Juventud. Santiago: Gobierno de Chile.

Issler, J. (2001). Embarazo en la Adolescencia. Revista de Posgrado de la Cátedra $V I^{a}$ Medicina, 107, 11-23. Obtenido el 14 de abril de 2009 desde http:// www.med.unne.edu.ar/revista/revista107/emb_adolescencia.html

Lamas, M. (s/f). La perspectiva de género. Revista de Educación y Cultura de la sección 47 del SNTE. Obtenido el 21 de abril de 2009 desde http://www.catedradh.unesco.unam.mx/webmujeres/biblioteca/Genero/LA\%20PERSPECTIVA\%20DE\%20GeNERO.pdf

Madrid, S. \& Olavarría, J. (2005). Sexualidad, fecundidad y paternidad en varones adolescentes en América Latina y el Caribe. UNFPA - Equipo de Apoyo Técnico para América Latina y el Caribe. Obtenido el 28 de abril de 2009 desde http://lac.unfpa.org/public/cache/offonce/pi $\mathrm{d} / 851$; jsessionid=C832284C9FD795A70EEA9B3DB39B8907

Olavarría, J. (2001). Y todos querían ser (buenos) padres. Varones de Santiago de Chile en conflicto. Santiago: FLACSO.

Olavarría, J. (2006). Estudio de la situación de maternidad y paternidad en el sistema educativo chileno. FLACSO-Chile. Obtenido el 20 de abril de 2009 desde http://www.mineduc.cl/biblio/documento/200612221242340. Informe\%20Final\%20Madres\%20Padres\%202006.pdf

Sandoval, C. (1996). Investigación cualitativa. Obtenido el 22 de abril de 2011 desde http://aprendeenlinea.udea.edu.co/revistas/index.php ceo/article/viewFile/1567/1223

Sandoval, M. (2006). La violencia escolar desde la teoría del riesgo y el cambio cultural. Investigaciones CEJU. Centro de Estudios en Juventud UCSH. Obtenido el 20 de diciembre de 2009 desde http://bibliotecavirtual.clacso.org.ar/ar/libros/chile/ceju/violencia.doc

Szil, P. (2007). Masculinidad y paternidad. Del poder al cuidado. Obtenido el 20 de enero de 2011 desde http://www.szil.info/uploads/documents/Spain/masculinidad_paternidad.pdf

Viveros, M. (2000). Paternidades y masculinidades en el contexto colombiano contemporáneo, perspectivas teóricas y analíticas. En N. Fuller (Ed.), Paternidades en América Latina, pp. 91-127. Lima: Pontificia Universidad Católica del Perú, Fondo Editorial.

Fecha de recepción: 03 de agosto de 2010.

Fecha de aceptación: 06 de junio de 2011. 
